\title{
3D multiscale hybrid modeling and simulation of vascular tumour growth including spatio-temporal distribution of central metabolism
}

\author{
M. Reuss*, A. Lapin \\ Stuttgart Research Center Systems Biology, University Stuttgart Germany \\ *e-mail:reuss@srcsb.uni-stuttgart.de
}

Key words: modeling, metabolism, cancer, tumour, cellular automaton, 3D multiscale model

Multiscale modeling and simulation in systems medicine is an emerging methodology and discipline to tackle the enormous challenges posed by complex diseases. Cancer modeling is an outstanding and important examples for solving problems which have important features at multiscale of time and space. The presentation aims at the application of a 3D hybrid multiscale hybrid discrete-continuum model to simulate angiogenesis and vascular tumour growth [1].The models is based on a cellular automaton approach and couples intracellular processes, active cell movement, cell-cell interaction, extracellular diffusion, an a dynamically evolving vascular network. For use of FDFG-PET data further extension of the model regarding the glucose balance are required. Coupling a dynamic model of the central metabolism in Hepatoma cells developed in our lab [2] which has been experimentally verified by quantitative measurements of metabolites and dynamic C13 flux analysis permits for the first time to consider a spatio-temporal distribution of intracellular central metabolism within a tumour.

\section{References}

1. Perfahl H., Byrne H., Chen T., Estrella V., Alarcon T., Lapin A., Gatenby R.A., Gillies R.J., Lloyd M.C., Maini P.K., Reuss M., Owen M.R. (2011) Multiscale modelling of vascular tumour growth in 3D: the role of domain size and boundary conditions. PLoS One. 6:e14790.

2. Maier K., Hofmann U., Reuss M., Mauch K. (2010) Dynamics and control of the central carbon metabolism in hepatoma cells. BMC Systems Biology. 4:54. 